
zonafranca
Gesty:
CENTRO DE ESTUDIOS
INTERDISCIPLINARIOS SOBRE MUJERES
I1. MAESTRIA "PODER Y SOCIEDAD

\title{
La perspectiva de género en la formación docente. Un espacio de des/encuentro.
}

Patricia Chantefort ${ }^{*}$

Federica Scherbosky**

\section{Resumen}

Existen hoy debates urgentes en torno a la pregunta por la relación entre género -o géneros- y educación. Desde nuestro lugar de formadoras de docentes proponemos diversos espacios para interrogarnos y reflexionar acerca de algunas de estas cuestiones que se nos tornan urgentes.

En esta dirección desarrollamos talleres en la Facultad de Educación de la Universidad Nacional de Cuyo en los que nos planteamos preguntas que, junto a nuestras estudiantes, consideramos imprescindibles para propiciar la deconstrucción y desarticulación de estereotipos de género sostenidos, tácita o explícitamente en cada texto escolar, en los juegos dentro y fuera del aula, en las expectativas de docentes, entre otros. Generamos espacios de taller para revisar y producir actividades áulicas con perspectiva de género. Desde este espacio más "práctico" pudimos revisar muchos supuestos y lograr inquietudes que no estaban puestas en juego.

El marco teórico que nos orienta se sostiene en los aportes de los feminismos, en sus afirmaciones más amplias. Problematizamos la noción de género y sexualidad a partir de los feminismos decoloniales y retomamos a los feminismos populares para pensar prácticas educativas emancipadoras.

\footnotetext{
* Facultad de Educación, Universidad Nacional de Cuyo. Contacto: patriciachantefort@gmail.com

** Instituto de Ciencias Humanas, Sociales y Ambientales, Centro Científico Tecnológico de Mendoza, Consejo Nacional de Investigaciones Científicas y Técnicas. Facultad de Educación, Universidad Nacional de Cuyo. Contacto: fedescherbo@gmail.com
}

Chantefort, Patricia; Scherbosky, Federica. "La perspectiva de género en la formación docente. Un espacio de des/encuentro" en Zona Franca. Revista del Centro de estudios Interdisciplinario sobre las Mujeres, y de la Maestría poder y sociedad desde la problemática de Género, №28, 2020 pp. 237-269. ISSN, 2545-6504 Recibido: 31 de julio 2020; Aceptado: 12 de noviembre 2020

Revista Zona Franca- Centro de estudios interdisciplinario sobre las mujeres (CEIM)- Maestría poder y sociedad desde la problemática de género (MG), Rosario, Argentina. ISSN, 2545-6504 http://zonafranca.unr.edu.ar/index.php/ZonaFrancal Número 28 (2020). 
Palabras clave: formación docente - género - educación feminismos

The gender perspective in teacher training. A space for dis-meetings.

\section{Abstract}

There are urgent discussions today about the question of the relationship between gender - or genders - and education. From our place as teacher trainers, we propose various spaces for questioning and reflecting on some of the questions that are urgent for us.

In this direction, we have developed workshops at Facultad de Educación Universidad Nacional de Cuyo in which we have posed questions that, together with our students, we consider essential to foster the deconstruction and dismantling of gender stereotypes that are sustained, either tacitly or explicitly, in eachschooltext, in games inside and outside the classroom, in teachers' expectations, among others. We generate workshop spaces to review and produce classroom activities with gender's perspective. From this more "practical" space we were able to review many assumptions and achieve concerns that were not put into play.

The theoretical framework that guide us is based on the contributions of feminists in their broader statements. We problematized the notion of gender and sexuality based on decolonial feminisms and took up popular feminisms to think about emancipatory educational practices.

Keywords: teacher training -gender - education - feminisms

\section{Introducción o acerca de cómo abrir espacios.}

Desde nuestro lugar de formadoras de docentes, en la Facultad de Educación de la Universidad Nacional de Cuyo, hemos propuesto en los últimos años espacios para interrogar e interrogarnos en relación con el género/los géneros, ya que consideramos se ha tornado en una instancia de urgente reflexión y abordaje con otras y otros. En nuestra experiencia de "formadoras" de futuras y futuros docentes de niveles inicial, primario y de personas con discapacidad hemos encontrado que la problematización del tema de los géneros, si bien imprescindible, no ha tenido -por lo menos hasta ahora- el lugar que debería, es decir, la problematización y el abordaje de algunas cuestiones básicas que nos

Revista Zona Franca- Centro de estudios interdisciplinario sobre las mujeres (CEIM)- Maestría poder y sociedad desde la problemática de género (MG), Rosario, Argentina. ISSN, 2545-6504 http://zonafranca.unr.edu.ar/index.php/ZonaFrancal Número 28 (2020). 
interpelan cada vez más hondamente en nuestro ser sujetos, mujeres, docentes. A modo de diagnóstico, encontramos que pocos espacios curriculares de la Facultad de Educación de la Universidad Nacional de Cuyo consideran la perspectiva de género, a la hora de elaborar sus contenidos.

Fruto de estas inquietudes y recorridos presentamos un proyecto de investigación, en el marco de las convocatorias de la Secretaría de Investigación, Internacionales y Posgrado de la Universidad Nacional de Cuyo titulado "Supuestos teórico-prácticos en la perspectiva de género en la educación. Un análisis situado en la formación docente de la Facultad de Educación". En este, por decirlo muy sintéticamente, sostenemos que la perspectiva de género en referencia a los estudios teóricos adoptados para una investigación, capacitación o desarrollo de políticas o programas implicaría: a) reconocer las relaciones de poder que se dan entre los géneros, en general favorables a los varones como grupo social y discriminatorias para las mujeres y sujetos feminizados/minorizados; b) que estas relaciones han sido constituidas social e históricamente y son constitutivas de las personas; y c) que atraviesan todo el entramado social y se articulan con otras relaciones sociales como las de clase, etnia, edad, preferencia sexual, por ejemplo.

El objetivo general del mencionado Proyecto de Investigación tiene que ver con indagar los supuestos teórico-prácticos presentes en la formación docente, particularmente en la Facultad de Educación de la Universidad Nacional de Cuyo, en relación a la ausencia y/o presencia de la perspectiva de género según los abordajes que hemos explicitado. Buscamos generar espacios para propiciar una perspectiva de género desde una mirada crítica y situada en Nuestramérica, con el propósito de deconstruir presupuestos y prejuicios presentes en posicionamientos clásicos, tradicionales y conservadores que funcionan en detrimento del respeto por los Derechos Humanos fundamentales.

Revista Zona Franca- Centro de estudios interdisciplinario sobre las mujeres (CEIM)- Maestría poder y sociedad desde la problemática de género (MG), Rosario, Argentina. ISSN, 2545-6504 http://zonafranca.unr.edu.ar/index.php/ZonaFranca| Número 28 (2020). 
Sabemos que quienes hoy son nuestras y nuestros estudiantes pronto se encontrarán en sus lugares de trabajo, frente al aula, con niños, niñas y niñes que -como corresponde a sus edades y los tiempos que vivimos- quizás tengan más necesidad de plantear y visibilizar temas relacionados con los géneros y las sexualidades de lo que lo tienen o pueden la mayoría de nuestras y nuestros estudiantes; y que consideramos, al menos en parte, es nuestra responsabilidad propiciar. No se trata simplemente de querer, o no, plantearlo, sino de que buena parte de la formación docente, sigue "domesticada" según las lógicas imperantes del patriarcado y el colonialismo. Pareciera que somos las y los adultos, las y los que tenemos más carga de prejuicios y posiciones más tradicionales, por lo que nuestro trabajo de comprensión del anquilosamiento de las categorías aprendidas es el primer paso para poder abordar y entender nuevas y muy acuciantes cuestiones y es nuestra obligación hacernos cargo de ello para poder llevarlo a nuestras y nuestros estudiantes.

En ese sentido es que venimos desarrollando talleres en la Facultad de Educación en los que nos planteamos preguntas que, junto a nuestras y nuestros alumnos, consideramos imprescindibles para propiciar la deconstrucción y desarticulación de estereotipos de género sostenidos -tácita o explícitamente- en cada texto escolar, en los juegos dentro y fuera del aula, en las expectativas de docentes, entre otros. Han sido variados los espacios en los que pretendemos junto con las otras y los otros, revisar, especialmente, nuestros aprendizajes provenientes del sentido común para desandarlo y concebir nuevas formas de pensarnos en nuestro ser sujetos generizados. Hemos intentado también producir actividades áulicas con perspectiva de género. Se trata entonces no solo de revisar y desandar supuestos teóricos, sino de visualizar cómo eso se hace patente en nuestras propias prácticas y así poco a poco intentar modificarlas.

En cada actividad, con modalidad de taller, que hemos llevado a cabo comenzamos con algunas preguntas que muchas veces por parecer obvias 0 sobreentendidas se asumen como resueltas. Por ejemplo: ¿Has percibido que tu

Revista Zona Franca- Centro de estudios interdisciplinario sobre las mujeres (CEIM)- Maestría poder y sociedad desde la problemática de género (MG), Rosario, Argentina. ISSN, 2545-6504 http://zonafranca.unr.edu.ar/index.php/ZonaFrancal Número 28 (2020). 
rol en la sociedad ha sido impuesto o construido por vos mismo/a? ¿En qué momento o momentos de tu vida ubicás la autopercepción de tu rol de género?, ¿Influyó el proceso de socialización en la educación en ese rol social? ¿Has percibido cambios en tus concepciones a partir del conocimiento de los procesos y debates de los feminismos como las concentraciones por "\#Ni una menos" y los paros del \#8M? ¿Sentís que has reproducido o cuestionado y transformado tu rol social? ¿Cuáles son o fueron las consecuencias de tu rol social actual?

A partir de estos interrogantes pretendemos desandar los preconceptos y prejuicios incorporados, la mayor parte de las veces, desde una concepción de género que considera a la diferencia como anormalidad y que asume una diferenciación jerárquica de los géneros como natural. Instancias estas que sin duda se perpetúan en el aula y que es parte de nuestra apuesta modificar.

\section{Algunos aportes feministas que nos sostienen en la apertura de}

\section{espacios.}

La cuestión del género tiene múltiples aristas y caminos posibles de ser transitados. En nuestro proceso de aprendizaje para repensar las identidades "de sentido común" reforzadas por el mundo escolar los planteamientos de Judith Butler han sido de gran ayuda. Aun a sabiendas de que habría otras posibilidades, sus aportes nos permitieron pensar la identidad como construcción.

Siguiendo a la mencionada autora y su teoría de la performatividad del género se puede afirmar que la reiteración de discursos y prácticas en relación con las sexualidades y los géneros -en nuestro caso en la escuela- produce cuerpos e identidades desde la norma heterosexual. A través de esta reiteración de prácticas normadas de género se consigue que los cuerpos e identidades sean vistos como un hecho natural y no como producción social, cultural e histórica.

Revista Zona Franca- Centro de estudios interdisciplinario sobre las mujeres (CEIM)- Maestría poder y sociedad desde la problemática de género (MG), Rosario, Argentina. ISSN, 2545-6504 http://zonafranca.unr.edu.ar/index.php/ZonaFranca| Número 28 (2020). 
En El género en disputa. El feminismo y la subversión de la identidad, Butler lleva adelante los primeros desarrollos de su teoría sobre el género, el sexo y la identidad sexual. ¿Cuáles son los principales aportes de este libro? Butler en primer lugar se introduce en la discusión entre esencialismo y constructivismo en materia de género, planteando una postura que trasciende ambas perspectivas. En segundo lugar, confronta y discute con el sistema de sexo/género, realiza luego una crítica anti-esencialista al sujeto político del feminismo y, por último, postula su noción del género como un acto performativo, entre otras cuestiones. El título del libro aporta, también, líneas posibles para pensar sus vínculos con lo queer.

La autora, en la obra mencionada, pretende trascender el debate del esencialismo y el construccionismo en materia de género. No se trata de que un enfoque construccionista, bajo una ley cultural inevitable, reemplace el paradigma esencialista, no es pasar de una construcción de género donde la biología es destino a otra donde lo cultural lo es. Justamente, la idea de Butler es romper con esta polaridad binaria. La revisión de las categorías de sexo y género sirve para romper con la representación dicotómica de sexo como destino biológico y de género como destino cultural. En realidad, decir que existe un sexo anatómico anterior a la idea cultural de género es también una construcción propia del sistema social que binariza el género. Según la autora, no hay una materialidad previa a la que se pueda acceder desde el orden del discurso, porque cuando se llega a ella ya estamos insertos en lo discursivo en tanto somos seres atravesados por el lenguaje. Pero tampoco se trata de afirmar que el cuerpo no es nada más que el producto de una construcción. Hay un nivel de la materialidad al que no se puede acceder. Butler toma distancia del construccionismo radical en el modo en que replantea la noción de sujeto, intentando no caer en el esencialismo ni en la ley cultural construccionista. En ese sentido, la opción que plantea para escapar a los límites del paradigma construccionista es la teoría performativa del género.

Revista Zona Franca- Centro de estudios interdisciplinario sobre las mujeres (CEIM)- Maestría poder y sociedad desde la problemática de género (MG), Rosario, Argentina. ISSN, 2545-6504 http://zonafranca.unr.edu.ar/index.php/ZonaFrancal Número 28 (2020). 
Según Butler, los géneros son ficciones reguladoras que se encargan de ubicar a los seres en un sistema binario de género, conceder sexualidades normalizadas y colocar a otras posibilidades abyectas en el lugar del otro, fuera de la sexualidad hegemónica y heterosexual. Para ella, los géneros no son verdaderos ni falsos, sino que se crean como los efectos de verdad de un discurso de identidad primaria y estable. $Y$ esta verdad es una invención, por lo tanto, un género verdadero es una fantasía instaurada en los cuerpos.

Si se acepta que la socialización escolar constituye un importante proceso de generización se comprende que el género opera como un significante de distribución social binaria y jerárquica de potencialidades, expectativas, atributos desarrollados por los seres humanos. Ni simple remisión al reino de la naturaleza, ni pura determinación sociocultural y tampoco mera creación de una individualidad singular. Contra esa supuesta naturaleza es que debemos encarar nuestros esfuerzos, para dejar de pensar en términos dicotómicos, para dejar de pensar las niñeces como sujetos pasivos y maleables.

En relación con los roles atribuidos y conformados es ineludible plantear una desencialización de las identidades. $Y$ esto traza otras cuestiones que deben problematizarse: por ejemplo, que las categorías sexuales mismas son menos estables y unificadas de lo que se piensa, que la identidad sexual puede ser experimentada como transitiva y discontinua, que la supuesta estabilidad de la identidad sexual es un proceso discontinuo que depende de contextos y prácticas sociales particulares y, por tanto, que los criterios de membresía a las categorías sexuales pueden y deben ser debatidos; y la escuela es un lugar ineludible para ello.

Hay quienes le han objetado a Judith Butler un supuesto desconocimiento de la materialidad de los cuerpos generizados. Creemos que en Cuerpos que importan aún más que en El género en disputa se aboca a desentrañar el asunto. De hecho, en el Prefacio de esta obra afirma que “... (se dio) cuenta de que no

Revista Zona Franca- Centro de estudios interdisciplinario sobre las mujeres (CEIM)- Maestría poder y sociedad desde la problemática de género (MG), Rosario, Argentina. ISSN, 2545-6504 http://zonafranca.unr.edu.ar/index.php/ZonaFranca| Número 28 (2020). 
podía fijar los cuerpos como simples objetos de pensamiento. Los cuerpos no solo tienden a indicar un mundo que está más allá de ellos mismos; ese movimiento que supera sus propios límites, un movimiento fronterizo en sí mismo, parece ser imprescindible para establecer lo que los cuerpos "son"' (Butler 2002, p. 11). Ni objetos de pensamiento ni pura materialidad, ya que hay algo que excede la mera materialidad del cuerpo, esa instancia de movimiento fronterizo que conecta al cuerpo como pura materialidad -como si tal cosa fuera posible- con su mundo.

Según Judith Butler, entonces, con su teoría de la performatividad, podemos insistir en que es la misma reiteración de discursos y prácticas constantes la que configura sexualidades y géneros en la producción de cuerpos e identidades desde la norma heterosexual binaria e imperativa. Por medio de esta repetición de normas de género se consigue que los cuerpos e identidades que estos asumen sean vistos como un hecho natural y no como producción social, cultural e histórica. Por ello para intentar una crítica de esos roles instituidos y pensando en la posibilidad de la existencia de subjetividades más autónomas es que sostenemos este desafío a partir de dos líneas de trabajo. Por una parte, buscamos analizar a través de qué estrategias se consolida en la pedagogía cotidiana la consideración del cuerpo como naturaleza pre-discursiva, sin significaciones previas, sin andamiaje cultural, lo que perpetúa la relación un cuerpo/un género/una sexualidad normal. Y, por otra, indagar acerca de cómo los movimientos sexo-genéricos y los distintos movimientos sociales relacionados con los feminismos y los enfoques de las y los disidentes sexuales piensan la cultura, la educación, la política y sus derechos. Si no se da cabida a estas subjetividades discordantes con la heteronormatividad y la moral religiosa, y a los saberes que ellos aportan como experiencias, es porque pretenden negarse otras subjetividades y otros saberes y es posible que no se esté dispuesto a conocer cualquier manifestación de otredad ni cualquier otro sujeto diverso que ponga en cuestión al disciplinamiento pedagógico.

Revista Zona Franca- Centro de estudios interdisciplinario sobre las mujeres (CEIM)- Maestría poder y sociedad desde la problemática de género (MG), Rosario, Argentina. ISSN, 2545-6504 http://zonafranca.unr.edu.ar/index.php/ZonaFranca| Número 28 (2020). 
Es imprescindible, además, un análisis del fenómeno de la educación sentimental diferencial por géneros que se expresa en múltiples dimensiones de la cotidianeidad escolar: en la asignación de distintos roles dentro del aula, en los cuentos y las lecturas, en los juegos, en el lenguaje, en la separación por asignaturas, etc. que tienden a relegar a las mujeres al extremo identificado con la debilidad, la pasividad, la dependencia, la motricidad fina, la delicadeza, mientras que desarrollan en los varones capacidades vinculadas a ser independientes, tener iniciativa, personalidad, gusto por la aventura, todas cuestiones importantes para la vida productiva y esenciales para promover liderazgo. Es en ese sentido que se ha postulado la idea de "dispositivos pedagógicos de género" para dar cuenta de cómo las formas de interacción, los roles, los formatos de participación, los juegos, los usos del lenguaje, las rutinas del espacio, etc., construyen interpretaciones y clasificaciones idealizadas y esencialistas respecto a lo que es ser mujer y ser varón con base en categorías de oposición jerárquica. Estos dispositivos actúan en el plano de los imaginarios, de las interacciones y en el plano material, que derivan en formas concretas de subjetivaciones de género (Morgade 2001).

Otras propuestas teóricas que hemos asumido, además de la teoría performativa de los géneros de Judith Butler, es el Feminismo Decolonial en sus afirmaciones más amplias, por ejemplo, en María Lugones y los aportes de los feminismos populares -a los que escuetamente nos hemos referido con Claudia Korol, por ejemplo-, a partir de los que se pretende pensar una concepción del género obviamente construida, pero, además, no necesariamente binaria. También acudimos a los trabajos de algunas especialistas argentinas que han profundizado en la relación entre educación y género, como la mencionada Graciela Morgade.

Se sabe que a estas alturas no podemos ya hablar de un feminismo sino de muchos, pues cada uno presenta una perspectiva distinta -y muchas veces en debate con otras- acerca de categorías fundamentales tales como: mujer/mujeres;

Revista Zona Franca- Centro de estudios interdisciplinario sobre las mujeres (CEIM)- Maestría poder y sociedad desde la problemática de género (MG), Rosario, Argentina. ISSN, 2545-6504 http://zonafranca.unr.edu.ar/index.php/ZonaFranca| Número 28 (2020). 
sexo biológico/género construido en correlación con el debate esencia/construcción 0 , más precisamente, naturaleza/cultura; binarismo de géneros/diversidad de géneros; heteropatriarcado entre otras.

Decidimos incorporarlos aportes de Lugones (2008) porque consideramos que da cuenta-asumiendo anteriores debates- de una posición situada. Pensamos lo situado para reflexionar desde nuestro contexto, no solo en términos nacionales sino sobre todo regionales, y en ese sentido elegimos hacerlo bajo la categoría e historia de Nuestramérica. Si bien junto con Martí apelamos a la emancipación que este concepto evoca, no podemos dejar de visibilizar la colonialidad ínsita que también presenta. Colonialidad que postula Aníbal Quijano como estructura de poder que se establece en el inicio de la modernidad con la conquista de América, pero que sigue presente en la actualidad. Lugones retoma la colonialidad propuesta por el pensador peruano para vincularla con el género, ya que sostiene que el sistema de género tal como lo conocemos se estructura en la modernidad con la consecuente colonialidad que esta presenta. Se hace necesario entonces establecer una interseccionalidad, un cruce, entre raza, clase, géneros y sexualidades. Es decir, comprender que no podemos referirnos a los géneros y las sexualidades en nuestro contexto geográfico e histórico particular si no asumimos que somos un continente, una nación que ha pasado por el proceso de colonización, proceso que dolorosamente hay que afirmar no es cuestión solo del pasado y ahí aparece la colonialidad como la constitución de subjetividades colonizadas, más allá de las supuestas independencias nacionales. Sin embargo, cabe aclarar que no se trata de una situacionalidad que nos cabe solo a nosotras/os por ser americanas/os, sino a todas las personas porque no hay conformación de la subjetividad que no sea situada y que en esta situacionalidad no se entrecruce la raza, la clase, las sexualidades y los géneros. En este sentido se conforma como una propuesta crítica hacia los feminismos occidentales hegemónicos que parece que han obviado aquella interseccionalidad y que de algún modo siguen bajo los preceptos modernos universalistas.

Revista Zona Franca- Centro de estudios interdisciplinario sobre las mujeres (CEIM)- Maestría poder y sociedad desde la problemática de género (MG), Rosario, Argentina. ISSN, 2545-6504 http://zonafranca.unr.edu.ar/index.php/ZonaFranca| Número 28 (2020).

Página 246 
Es imperioso asumir este cruce para poder desde allí generar prácticas liberadoras. Se trata de entender el patriarcado desde la colonialidad del género pensando cómo se construyó el poder global actual desde lo que Lugones denomina Sistema Moderno/Colonial de Género. Podría sostenerse que, ante la necesidad en apariencia natural de la clasificación, el género como atributo se gesta como dispositivo de control en la matriz semiótica de una tradición con fuerte disposición binaria y jerárquica. No puede negarse la pre-existencia de la disposición hacia las divisiones sociales basadas en los géneros, pero debe comprenderse que su lógica dicotómica y su asimetría simbólica y política tienen un claro anclaje en la tradición europea moderna, que conformó a la par la configuración del otro no-europeo. Es en la matriz comprensiva de la existencia binaria en que uno de los términos se resuelve como prioritario, superior o sencillamente mejor y se resignifican el varón y la mujer como estereotipos sociales fijos.

Este modo de la configuración de la experiencia según patrones de poder modernos/coloniales está así formulado a partir de grandes matrices simbólicas europeas. En primer lugar, la comprensión de lo real como compuesta por términos opuestos y excluyentes, aplicables a la totalidad de la experiencia humana de todos los pueblos del mundo y, por otra parte, el conjunto de sistemas jerárquicos funcionales a la colonización. Lo que en un momento actuara como instrumento para la hegemonía con consecuencias político-jurídicas se prolongó, luego, como una reedición de la colonización simbólica que no cede terreno ideológico, ni se disipa, aun habiendo transcurrido los movimientos de independencia de las naciones, que se plantean desde esta perspectiva como formales y nominales, ya que las subjetividades siguen colonizadas.

La formación del mundo cristiano-centrado/occidental-centrado, modernocolonial y capitalista-patriarcal propuso un número de bloques jerárquicos indispensables para la dominación, entre los que se encuentra una jerarquía patriarcal global con sus respectivas relaciones de poder a nivel de géneros y de

Revista Zona Franca- Centro de estudios interdisciplinario sobre las mujeres (CEIM)- Maestría poder y sociedad desde la problemática de género (MG), Rosario, Argentina. ISSN, 2545-6504 http://zonafranca.unr.edu.ar/index.php/ZonaFranca| Número 28 (2020). 
sexualidades. La colonialidad, entonces, debe ser entendida como un elemento constitutivo y específico del patrón mundial del poder capitalista, que se origina y mundializa a partir del "descubrimiento/encubrimiento" de América. La colonialidad, como contracara ineludible de la modernidad, implica la imposición de una clasificación racial, de clase, de sexualidad y de género para la población del mundo que opera en cada uno de los planos, ámbitos y dimensiones materiales y subjetivas de la existencia cotidiana.

A partir de los aportes de Lugones en términos de una conciencia histórica y situada de nuestra propia subjetividad, es que asumimos los planteos teóricoprácticos de los llamados feminismos populares. En sus múltiples vertientes se plantean como críticos del sistema moderno-colonial patriarcal, blanco y heterosexuado. $Y$ en este mismo sentido nos habilitan la posibilidad de pensar otra educación posible, otra escuela posible para las subjetividades múltiples que hoy patentizan demandas que la escuela moderna que tenemos no resuelve. Claudia Korol introduce estos feminismos populares afirmando:

\footnotetext{
“...hay un movimiento que crece como conciencia histórica, que se "encuerpa" desde la memoria y cambia -nos cambia- la vida cotidiana, los modos de estar en el mundo, de ser y de creer. Son colectivos que se van multiplicando entre las mujeres y las disidencias sexuales, que asumen el feminismo como un modo de desafiar las múltiples opresiones producidas por el capitalismo colonial y patriarcal. [...] Feminismos de sujetas no sujetadas, que se organizan para responder colectivamente a los desafíos de la sobrevivencia. No son un relato para entendidas, sino una práctica rebelde, y una teoría que se amasa en los comedores populares." (Korol 2016, p. 15-16).
}

Claro que estas prácticas militantes y este modo de ver el mundo no es algo que se aprende sin más, no es posible adentrarse en estos feminismos si no nos ponemos, en primer lugar, en situación genuina de aprender de aquellas que han vivido y viven en contacto con la tierra, de la tierra, que saben en los cuerpos, en

Revista Zona Franca- Centro de estudios interdisciplinario sobre las mujeres (CEIM)- Maestría poder y sociedad desde la problemática de género (MG), Rosario, Argentina. ISSN, 2545-6504 http://zonafranca.unr.edu.ar/index.php/ZonaFranca| Número 28 (2020). 
las cuerpas que nada es posible si no se hace en comunidad, entre las personas pero también en contacto respetuoso con la naturaleza.

Es muy difícil para quienes nos hemos formado y transitamos el mundo académico, atravesado por el eurocentrismo y la consiguiente mirada de la modernidad/colonialidad, ponernos en un lugar respetuoso y con auténticos deseos de aprender junto a nuestras y nuestros estudiantes, ya no disciplinadamente ni tratando de ver en qué posición de las jerarquías establecidas nos colocamos. Es otro el camino, es la escucha, es la solidaridad, es la sororidad. Imaginar y crear una pedagogía feminista desde esta perspectiva es un desafío para nosotras que pretendemos pensar en otro modo de enseñar tanto como de aprender y ello no es fácil.

"Lo difícil, es asumir el cambio que implica en las conductas de cada uno y cada una. Porque el orden verticalista y autoritario es tranquilizador para los de arriba, pero también para Ixs de abajo [...] Por eso, la pedagogía feminista asume la dimensión grupal como una necesidad básica para que los dolores que produce el desaprendizaje de las opresiones, puedan ser compartidos y sostenidos en los colectivos. También busca una interpelación sistemática entre teoría y práctica, que permita leer las experiencias individuales y sociales, y escribir nuestras historias con un horizonte emancipador" (Korol 2016, p. 22-23).

Esa pedagogía feminista pretende ordenar de otro modo saberes y prácticas, conocimientos y experiencias, razón y emoción. Dicha pedagogía recupera de la educación popular datos centrales como el lugar del cuerpo en el proceso educativo, la dimensión lúdica, la educación por el arte, el teatro de los y las oprimidas, la danza, el canto, y el diálogo desde diversas perspectivas ideológicas emancipatorias tal como pueden ser los ecofeminismos, los feminismos marxistas, los feminismos negros, indígenas, lésbicos, etc.

La propuesta y los objetivos de una pedagogía feminista no son fáciles de instalar en un sistema educativo tan cerrado, disciplinador, controlador,

Revista Zona Franca- Centro de estudios interdisciplinario sobre las mujeres (CEIM)- Maestría poder y sociedad desde la problemática de género (MG), Rosario, Argentina. ISSN, 2545-6504 http://zonafranca.unr.edu.ar/index.php/ZonaFranca| Número 28 (2020). 
estructurado y normalizador como el que nos incluye pero, a pesar de ello, esa pedagogía se presenta como un importante ideal a llevar a cabo. $Y$ además es preciso tener en cuenta que justamente la pedagogía feminista se presenta como lo opuesto a lo tradicional en cuanto está en movimiento, reconstruyéndose siempre. Como dice Claudia Korol:

"La pedagogía feminista está rehaciéndose de modo permanente, en la medida en que cambian los desafíos, que somos otrxs los sujetos colectivos que la asumimos como parte de nuestra propuesta política. Hay un diálogo intergeneracional que nos ayuda a pensar que las huellas que dejamos, van creando nuevas posibilidades a las colectivas más jóvenes, para identificar las maneras propias de estar en el mundo." (Korol 2016, p. 23).

\section{El cuerpo utopía o acerca de cómo habilitar diversas corporalidades}

\section{en la escuela.}

Un fragmento de una entrevista a Diana Maffía realizada por Gabriela Navarra en el "Diario La Capital" de Rosario parece un buen disparador para empezar a pensar el lugar de la educación en la construcción de un mundo más justo e igualitario:

"Es que lo más trágico del patriarcado es que las mujeres, que somos sus
víctimas, seamos a la vez sus grandes transmisoras porque nos han
delegado la función reproductiva de cuidado, la primera educación. Es
una trampa perfecta. Las mujeres formamos las mentalidades, la
reproducción de los roles. Queremos que nuestros hijos se adapten para
ser felices. Si es gay o lesbiana van a tener problemas; igual que si el
varón no es fuerte y competitivo y la nena no juega con brillantitos. En
este modelo de felicidad, atado a la adaptación, el patriarcado, como todo
sistema autoritario, tiene dos botones: el de la persuasión y los premios si
te adaptás y el del castigo si no te adaptás. Es un modelo de silencio y de
aceptación para no incomodar que se sigue imponiendo. Por eso chocan

Revista Zona Franca- Centro de estudios interdisciplinario sobre las mujeres (CEIM)- Maestría poder y sociedad desde la problemática de género (MG), Rosario, Argentina. ISSN, 2545-6504 http://zonafranca.unr.edu.ar/index.php/ZonaFrancal Número 28 (2020). 
las pibas jóvenes que protestan, que se rebelan. Hay una generación que irrumpe de golpe en el feminismo y que no negocia..." (Maffía 2019).

Esta primera educación no sólo está asignada socialmente a las mujeres en tanto madres, sino también fuertemente a las mujeres en tanto maestras. Ahí pretendemos generar una incisión, un quiebre, o al menos una pregunta, para que las y los docentes que formamos en la Facultad de Educación podamos empezar a romper el círculo de lo dado, de aquello naturalizado y que como tal se perpetúa como lo obvio. Implica un cuestionamiento y un no adaptarse a la formación recibida, para poder luego también increpar y ampliar los sistemas escolares.

Un punto que consideramos preocupante es el lugar del cuerpo en la educación, ya que observamos cómo tanto en sus teorías, como en sus prácticas y en sus instituciones persiste un desconocimiento/ocultamiento del cuerpo de las niñas y los niños, de las y los jóvenes. En El cuerpo utópico Foucault sostiene que el cuerpo es ese espacio que, aunque se intente borrar, es "lugar irremediable" (Foucault 2010). Se ha dado tanta importancia a lo no corpóreo, a la mente, el espíritu, el alma que casi se ha logrado la pretensión del olvido del cuerpo: "Pero tal vez la más obstinada, la más poderosa de esas utopías por las cuales borramos la triste topología del cuerpo nos la suministra el gran mito del alma, desde el fondo de la historia occidental" (Foucault, 2010, p.12). Y, en relación con lo anterior nos interpela, aquí, una frase de este autor en la que sostiene que "el cuerpo humano es el actor principal de todas las utopías" (Foucault 2010, p.13)'.

Pero si es el cuerpo el lugar de la utopía, de lo por hacer, de los propósitos a lograr ¿qué cuerpos encontramos al relacionarnos con otros?, ¿son los cuerpos los desconocidos de nuestras historias individuales?, ¿cómo nos hemos referido a nuestros cuerpos en el descuido de ellos cuando priorizamos "el alma"? Y

\footnotetext{
${ }^{\prime}$ Retomamos el planteo de Foucault en su recuperación del cuerpo, en sentido amplio, y en el análisis genealógico que realiza acerca de la segregación del mismo. No obstante, no nos desentendemos de las críticas -feministas- al autor que señalan que si bien recupera el cuerpo no lo hace de manera generizada, lo que implica una gran omisión de las relaciones de poder en las que estos cuerpos se insertan y un olvido de los cuerpos feminizados, minorizados. Cfr. Federici ([2004] 2010).
}

Revista Zona Franca- Centro de estudios interdisciplinario sobre las mujeres (CEIM)- Maestría poder y sociedad desde la problemática de género (MG), Rosario, Argentina. ISSN, 2545-6504 http://zonafranca.unr.edu.ar/index.php/ZonaFrancal Número 28 (2020). 
entonces, cabe preguntarse cómo son "marcados", "nombrados", esos cuerpos que, por un lado, parecen ignorados y, por otro, constituyen aquello que se presenta como lo más visible e ineludible. Si al mismo tiempo se piensa con Graciela Morgade que "toda educación es sexual" (Morgade 2011) puede entenderse que no se trata de una contradicción cuerpo/alma y no es simple indiferencia ante el cuerpo. Es la historia occidental misma la que se manifiesta en esa distintiva oposición.

Y el cuerpo es sexualidad en todos los ámbitos y, claro está, en el espacio de la educación también. Esta autora sostiene, entonces que "La sexualidad estaba y está en todas partes porque es una dimensión de la construcción de la subjetividad que trasciende ampliamente el ejercicio de la genitalidad o una expresión de la intimidad" (Morgade, 2011, p. 10)

Cuando preguntamos qué se enseña, quién enseña, para qué se enseña, cómo se aprende y otros interrogantes propios de las teorías pedagógicas sostenemos y reafirmamos que el hecho de colocar en la periferia de dichos cuestionamientos al cuerpo no es casual ni es mera anécdota. Podría decirse que ha sido parte del núcleo de las teorías pedagógicas y las didácticas y se observa claramente qué es lo que está puesto en valor y por ende visibilizado y qué no. Junto con Morgade consideramos que:

\footnotetext{
"En este sentido, entender la "sexualidad" como campo de "contenidos a enseñar" despliega también diferentes apelaciones sobre aquello que es considerado valioso poner en juego en el contexto de la escuela y reedita en un nuevo contexto las ya viejas discusiones del campo pedagógico: la tensión entre "conocimiento" e "información", la tensión entre "saber" e "ignorancia", la tensión entre "el sujeto ideal" y el "sujeto real", la tensión entre universalidad y particularidad en las diferentes definiciones de justicia, y muchas más..." (Morgade 2011, p.23-24)
}

Este supuesto "olvido" del cuerpo -y la consiguiente sobrevaloración del "alma"- tiene objetivos muy precisos, aunque no explícitos. Es una estrategia de

Revista Zona Franca- Centro de estudios interdisciplinario sobre las mujeres (CEIM)- Maestría poder y sociedad desde la problemática de género (MG), Rosario, Argentina. ISSN, 2545-6504 http://zonafranca.unr.edu.ar/index.php/ZonaFrancal Número 28 (2020). 
control que se ha venido gestando en instituciones educativas "domesticadoras" y con pedagogías que obstruyen la posibilidad de elecciones libres para todas y todos. Es imprescindible pues, ocuparse de lo que se presenta obturado, que se comprenda que los cuerpos son cuerpos sexo-genéricos y que, por lo tanto, la educación, como instancia liberadora, debería eliminar toda actividad o tarea dirigida a poner límites a los cuerpos para encaminarlos por los carriles de la "normalidad" que distinguen lo femenino y lo masculino como si fueran esencias inmutables. Solo así podremos efectivamente sostener que cada cuerpo en libertad es el "lugar de la utopía". Y apostamos a que esa construcción deje de ser utópica para que se convierta en un topos concreto de emancipación para la multiplicidad de subjetividades posibles.

En cierto sentido todas y todos tenemos el hábito, la costumbre de clasificar, juzgar y categorizarlo todo. El problema no es la clasificación en sí, sino que esta trae consigo una cierta normalidad y aquello que no entre en esta categoría cae bajo la "anormalidad". La clasificación y la jerarquización de la misma se presentan de manera inescindible y usualmente bastante estanca. Consideramos que el respeto a la diferencia es una instancia, aunque sea de mínima, que nos permite empezar a pensar por fuera de la lógica binaria que solo permite dos identidades, donde la masculina es sobrevalorada en relación a la femenina, pero en la que además no hay otras identidades posibles. A partir de un mínimo respeto podemos empezar a visibilizar a otras identidades y así tomar en cuenta que cuando estamos frente a lo que usualmente se denomina "diferencia" estamos frente a una subjetividad única e irrepetible, a pesar de haber sido constituida sobre la base de repeticiones de actos que la fueron conformando. No pensar dicotómicamente es un compromiso ético con la diferencia, asumiéndonos nosotras y nosotros con la misma perspectiva, ya que no podemos pensar que la diferencia siempre es el otro, pues al apostar a la concepción de una subjetividad única e irrepetible no habría quien no sea diferente.

Revista Zona Franca- Centro de estudios interdisciplinario sobre las mujeres (CEIM)- Maestría poder y sociedad desde la problemática de género (MG), Rosario, Argentina. ISSN, 2545-6504 http://zonafranca.unr.edu.ar/index.php/ZonaFranca| Número 28 (2020). 
Una mirada deconstructivista permite pensar en el desplazamiento de las identidades de género y sexuales de su relación, supuestamente determinada, por "la naturaleza" dando lugar a nuevos y múltiples sexos, sexualidades y géneros que pueden desarticular las marcas del género y la sexualidad de las significaciones antes atribuidas al sexo biológico. Las categorías de género y sexualidad devienen terrenos abiertos a todo tipo de cuerpos y a variadas formas de significación. El cuerpo mismo es un campo abierto a diversas posibilidades interpretativas. Deja de ser entendido como ahistórico, como lo dado biológicamente, materia pasiva sobre la que se proyecta lo masculino y lo femenino. Esta mirada sospecha de la distinción sexo/género y tiende a trasgredir el dualismo mente/cuerpo.

Si los cuerpos por su diversidad inherente impiden su incorporación, su hacerse cuerpo, de acuerdo con un modelo singular universal, entonces las mismas formas que toma la subjetividad no son generalizables. La subjetividad no puede elaborarse conforme a los ideales universalistas del humanismo, no hay un concepto de lo "humano" que incluya a todos/as los/as sujetos sin violencia, sin olvido o de manera no residual. Además, esos cuerpos se constituyen en una subjetividad generizada siempre en relación con otros cuerpos que también poseen y adquieren distintas condiciones y representaciones simbólicas. E proceso de reconocimiento en términos de constitución conjunta con la alteridad es ineludible para repensarnos desde estas perspectivas no universalistas, ya que es en el "cara a cara" con la alteridad que nos conformamos como sujetos.

Pensar las cosas de otra manera es una tarea compleja, no se dispone siquiera de un lenguaje con el que poder referirse a una subjetividad corporizada que se resista al dualismo y que elabore alternativas a él. Son necesarias representaciones diferentes a las que han dominado la historia del pensamiento, se necesita pensar en el cuerpo como un campo plural, múltiple y abandonar aquel que funciona como el delegado o el representante de otros. Un campo como espacio discontinuo, no homogéneo, un espacio que admita las multiplicidades. En

Revista Zona Franca- Centro de estudios interdisciplinario sobre las mujeres (CEIM)- Maestría poder y sociedad desde la problemática de género (MG), Rosario, Argentina. ISSN, 2545-6504 http://zonafranca.unr.edu.ar/index.php/ZonaFranca| Número 28 (2020). 
vez de considerar, entonces, al sexo como acultural y prelingüístico -y en este sentido "natural"-, y al género como una categoría construida, la mirada deconstructivista se dirige a quebrantar la dicotomía. El cuerpo en sí es objeto político, social y cultural, no una naturaleza pasiva gobernada por la cultura. Y el cuerpo resiste como espacio de lo incierto, del cambio, del movimiento. Cada vez que se cree que el cuerpo ha sido capturado por las tecnologías de poder, éste se rebela y se recodifica, desafiando al poder que le ha dado forma pero que no logra otorgarle un único y permanente sentido. Resiste también en relación con los intentos de constituirlo en máquina eficiente, callada y obediente.

Si se mantiene la dicotomía sexo/natural - género/construido, este último se consolida como un complejo mecanismo que define al sujeto como masculino o femenino en un proceso de normalización y regulación orientado a producir al ser humano esperado. Construye así las mismas categorías que se propone explicar: varón, mujer, heterosexual, homosexual, siempre de acuerdo a la coincidencia o disidencia con lo "naturalmente" asignado en términos de sexo. Así, aunque se plantee como distanciado del sexo en términos de construcción social, no deja de ser una construcción normalizante. $Y$ una de las cuestiones centrales a deconstruir es, como bien decíamos antes, el binarismo. Ya no puede pensarse en términos binarios, como si existieran solo dos géneros que de algún modo coinciden o no con el sexo dado. Hay múltiples formas de autopercibirse que rompen esta dicotomía al presentarse como no binarie, o transgénero, entre otras.

Nos interesa destacar, particularmente, el relato y la vivencia de Gabriela Mansilla, quien es fundadora de la Asociación Civil Infancias Libres, a partir de la experiencia de su hija Luana, quien fue la primera niña trans en Argentina en obtener su Documento Nacional de Identidad con identidad femenina autopercibida, con solo 6 años". En una entrevista publicada por Página 12 nos

"Esta experiencia está relatada sobre todo en un libro que publica con el título Yo nena, yo princesa. (2014)

Revista Zona Franca- Centro de estudios interdisciplinario sobre las mujeres (CEIM)- Maestría poder y sociedad desde la problemática de género (MG), Rosario, Argentina. ISSN, 2545-6504 http://zonafranca.unr.edu.ar/index.php/ZonaFranca| Número 28 (2020). 
interpela directamente en esta tarea de formadoras de formadoras/es al afirmar: "Quiero que el cuerpo travesti y trans esté en la escuela" (Mansilla 2019)

Pero sin duda no se trata solo de una expresión de deseo, sino del reclamo en tanto derecho de aquellas personas que escapan al binarismo estanco que buscamos desandar. Se pronuncia crítica de la ley de Educación Sexual Integral porque esta no incluye la transgeneridad. Afirma que trabaja la diversidad, pero en relación con la homosexualidad y no así con lo travesti y lo transgénero. Ese reconocimiento sigue fuera del ámbito escolar, del proceso de enseñanza y de respeto. Siguen siendo cuerpos abyectos que no calzan en la norma, pero tampoco en aquello considerado diverso. Están simplemente por fuera. De allí su trabajo en la Asociación para acompañar a las familias y a las personas trans para poder transitar sus infancias de un modo más libre. Pero en este tránsito la escuela es un lugar ineludible, en el que usualmente se vive más opresión y discriminación que libertad y respeto. Al preguntarle acerca de su acompañamiento en relación con las escuelas responde:

No se puede generalizar, pero en la mayoría de los casos de niñeces trans que acompañé tuve que enviar una nota a sus escuelas exigiendo el cumplimiento de la ley de Identidad de Género y los pasos administrativos que deben seguir, porque los desconocen. No todos los pibes y pibas de la secundaria tienen hecho el DNI pero los deben nombrar con su nombre elegido. Algo muy importante es que si ellxs están pendientes del rechazo social, de los estereotipos que no alcanzan, tampoco puede rendir en la escuela como se espera. ¿Cómo quieren que se concentren en estudiar cuando tiene que concentrarse en sobrevivir? La clase de educación física es traumática para ellxs. (Mansilla 2019)

Son innumerables las estigmatizaciones, no sólo en la clase de educación física, en la utilización del baño, en la clase de biología, sino hasta el propio nombre. Gabriela propone un modo más unisex en el que las determinaciones estén menos presentes. Esto implica ya no pensar en "toallitas femeninas" sino en

Revista Zona Franca- Centro de estudios interdisciplinario sobre las mujeres (CEIM)- Maestría poder y sociedad desde la problemática de género (MG), Rosario, Argentina. ISSN, 2545-6504 http://zonafranca.unr.edu.ar/index.php/ZonaFranca| Número 28 (2020). 
apósitos higiénicos, ni tampoco en órganos sexuales masculinos o femeninos, sino de aparatos sexuales fecundantes o gestantes. Sostiene que hay que:

Dejar de asignarle género a los órganos y su función natural: entonces, podemos hablar de un sistema sexual fecundante, que es el que tienen Luana, y muchos varones cis de la clase, y de un sistema sexual gestante, que es el que tienen la mayoría de las compañeritas y puede tener cualquier varón transgénero. $Y$ con eso realmente está respetado y aceptado el cuerpo de mi hija dentro de su construcción identitaria en la escuela. Verlo en una carpeta y que todos los alumnos se llevaron eso a su casa es el resultado de mis seis años de lucha dentro de la misma escuela. Para mi es grandioso. (Mansilla 2019)

Este es el desafío que nos proponemos como formadoras de futuras/os docentes. Abrir un espacio para empezar a desnaturalizar eso que tenemos tan arraigado, pero que a su vez se presenta de manera urgente. Concordamos con la mamá de Luana en que la base de todo este cambio tan necesario es la educación, para que no sea solo producto de la lucha titánica de una madre para que el cuerpo de su hija sea aceptado y respetado. Abrimos distintas instancias de reflexión y praxis para que este cuerpo utópico, este lugar de la utopía sea cada vez menos utópico y más real. Por ese topos posible abogamos.

Asumimos lo escrito por la Colectiva Feminista La Revuelta en "Espacios escolares y relaciones de género", dentro del libro Feminismos populares, pedagogías y políticas, en el que sostienen que:

"Nosotras, maestras, profesoras, educadoras, blancas, morenas, heterosexuales, lesbianas, bisexuales...damos valor a lo personal. Lo hacemos político. $Y$ esto porque el compartir con otras mujeres las vivencias personales en nuestro paso por las instituciones educativas como estudiantes, como trabajadores de la educación, no podíamos aceptar que todo lo que teníamos en común fuese simple coincidencia. Entre las feministas las experiencias personales pueden ser materia prima, datos para la investigación, para la reflexión, para la producción de saberes y conocimientos" (Korol 2016, p. 103)

Revista Zona Franca- Centro de estudios interdisciplinario sobre las mujeres (CEIM)- Maestría poder y sociedad desde la problemática de género (MG), Rosario, Argentina. ISSN, 2545-6504 http://zonafranca.unr.edu.ar/index.php/ZonaFrancal Número 28 (2020). 
No podemos dejar de preguntarnos qué hay de común en estos tránsitos por las instituciones educativas. ¿Es la opresión de la que hablaba Gabriela Mansilla? ¿Es este sentido común en el que no todos los cuerpos caben, es esta normalidad que en el mejor de los casos podría aceptar respetuosamente la diversidad? ¿De qué diversidad hablamos? ¿Buscamos respeto o reconocimiento?

Estos son algunos de los interrogantes que nos vinculan de lleno con el mundo escolar.

\section{¿Qué pasa en la escuela? Instancias para repensar la perpetuación de lo dado.}

La escuela como buena institución moderna se encuentra, desde entonces, fuertemente involucrada en la producción y reproducción de un imaginario socialgenérico. Es la sexualidad el terreno político donde se impregna cierto sentido común "natural" en relación al "deber ser" varón o mujer, pero además legitima uno de esos modos de existencia por encima del otro. Instancias que cristalizan en el patriarcado.

En el escenario educativo, el discurso pedagógico realiza operaciones de clasificación, ordenamiento, normalización, normativización, regulación, regularización y produce un lenguaje escolar centrado en el silenciamiento de algunas voces y en la habilitación de otras. Se normalizan las operaciones de inclusión y exclusión como parte inherente al lenguaje escolar. En la vida cotidiana de la escuela esto se expresa en la negación y exclusión de lo femenino, por un lado, y, por otro, en una cierta visibilización de lo femenino, pero siempre aferrado a ciertos estereotipos producidos a lo largo de la historia que implican una subsunción de esta a lo masculino.

Volvemos a tomar palabras de la Colectiva Feminista La Revuelta para pensar con ella que:

Revista Zona Franca- Centro de estudios interdisciplinario sobre las mujeres (CEIM)- Maestría poder y sociedad desde la problemática de género (MG), Rosario, Argentina. ISSN, 2545-6504 http://zonafranca.unr.edu.ar/index.php/ZonaFranca| Número 28 (2020). 
"La escuela -heredera de la moderna sociedad occidental- es un espacio en que se producen subjetividades e identidades, mediante un proceso complejo, plural y permanente, en el cual las/os sujetas/os están implicadas/os y son activas/os participantes. El espacio escolar fue desde sus orígenes un campo instituidor de diferencias, aunque los discursos de los organismos y autoridades gubernamentales, de los medios de comunicación, de las leyes educativas, refieran con énfasis a la igualdad. La escuela marca, mediante mecanismos de clasificación, ordenamiento y jerarquización, las posibilidades o el destino de cada sujeta/o" (Korol 2016, p. 105).

Estas marcas no son sólo simbólicas o parte de un imaginario. Se hacen presentes, como bien afirma La Colectiva, en las posibilidades concretas de cada persona. Se hacen marca en el cuerpo sexuado y constituyen representaciones e identidades que son muy difíciles de modificar. Preguntarse por las representaciones que se tienen del propio cuerpo es preguntarse, al mismo tiempo, por cuestiones concernientes a la identidad. Si se acuerda en que el cuerpo no es la "carne" que existe fuera del imaginario social sino una especie de fabricación dependiente de las articulaciones y de las valoraciones predominantes, entonces los límites materiales y simbólicos del "yo" también son producciones sociales y, por lo tanto, objeto de las luchas por la transformación. El cuerpo, no como sede de la subjetividad -lo que implicaría seguir manteniendo la dualidad cuerpo-alma-, sino como la subjetividad misma, es una construcción continua, con cierta inestabilidad, en un devenir que incluye posibilidades de cambio. Aquí la noción de resistencia foucaultiana (2009) adquiere un gran potencial. Este es el cuerpo que proponemos repensar en la escuela y no aquel que se enquista en lo dado. $\mathrm{Y}$ es en este entrelazamiento de la escuela con los saberes y los cuerpos cuando lo que es propio de cada persona se convierte en una fuerza política. Por ello adherimos a lo que Claudia Korol sostiene en su artículo "Feminismos populares", al afirmar que:

Revista Zona Franca- Centro de estudios interdisciplinario sobre las mujeres (CEIM)- Maestría poder y sociedad desde la problemática de género (MG), Rosario, Argentina. ISSN, 2545-6504 http://zonafranca.unr.edu.ar/index.php/ZonaFrancal Número 28 (2020). 
"«Lo personal es político», decimos las feministas. Esto apela a las dimensiones pedagógicas y culturales de las revoluciones. Transformar los vínculos, saliendo del «sálvese quien pueda» para llegar al «vamos juntxs», dejar el «ordeno-mando-obedezco» para llegar al «decidimos juntxs y juntxs hacemos», es una tarea gigantesca que va a contramano de lo aprendido como jerarquías, criterios de autoridad, en los límites establecidos sobre la base del aturdimiento que producen los medios de comunicación masiva, el sistema educativo tradicional, la coerción social y la represión". (Korol 2016, p.150)

Este sistema educativo tradicional tiende a negar u obstaculizar un proceso relativamente autónomo de subjetivación a grandes sectores de la población al reducir sus opciones de identificación. Algunas de esas formas de discriminación están vinculadas con los géneros y las sexualidades y los efectos que las operaciones discursivas producen en las niñas, los niños y les niñes, las, los y les adolescentes, las, los y les jóvenes que transitan la institución escolar. También sabemos de la histórica segregación de las mujeres y sujetos feminizados de los ámbitos del saber, no sólo en términos del retraso en el derecho a la educación, sino también de la exclusión de sus producciones por el mero hecho de ser realizadas por mujeres. María Luisa Femenías recoge esa deuda en Ellas 10 pensaron antes y muestra la exclusión de las mujeres en el ámbito del pensamiento a lo largo de la historia. Por lo que al repensar la institución escolar y la reproducción de los saberes establecidos no podemos dejar de revisar estas relaciones de exclusión. Como bien nos comenta:

"Un problema no ajeno es que la organización del saber y su transmisión suele hacerse según pares dicotómicos opuestos regidos por la dupla varón/mujer. Estos pares no conservan un equilibrio valorativo y ponderan uno de sus lados, el masculino, sobre el que se moldea el conocimiento, como si fuera su eje ideal, único y modélico". (Femenías 2020, p. 25).

Nosotras como profesionales de la filosofía no podemos dejar de lado y sin cuestionar el sesgo sexista que todas las formas de pensamiento han tenido y que

Revista Zona Franca- Centro de estudios interdisciplinario sobre las mujeres (CEIM)- Maestría poder y sociedad desde la problemática de género (MG), Rosario, Argentina. ISSN, 2545-6504 http://zonafranca.unr.edu.ar/index.php/ZonaFrancal Número 28 (2020). 
se hace particularmente evidente en el ámbito de la filosofía. Desde el espacio curricular que compartimos, a saber, Antropología Filosófica, no podemos dejar de cuestionar la definición más inmediata que se da de la misma, en la que se repite casi sin pensar, que se trata de la pregunta filosófica por el hombre. Sin embargo, se nos hace imperioso preguntar ¿y las mujeres dónde estuvimos en la historia de la filosofía?, ¿no somos capaces de hacer filosofía porque como el imaginario social afirma "somos seres emocionales"?, ¿cuántas mujeres han sido excluidas de este espacio, desde Hipatia hasta nuestros días? Y en tanto pretendemos que nuestras y nuestros estudiantes formen una capacidad crítica debemos, como punto de partida, problematizar esta relación de lo femenino como aquello excluido de la producción de conocimiento. Por lo que compartimos la afirmación que realiza Femenías al sostener que: "La crítica más reciente ha mostrado que tanto la ciencia como la filosofía son prácticas culturales que se suman a la construcción de un imaginario social generizado, según una compleja red de valoraciones organizadas jerárquicamente, que se sostienen unas a otras" (Femenías 2020, p. 25). Por lo cual acompañamos los interrogantes que plantea más adelante en los que afirma:
“¿no deberíamos reconocer ya la importancia del sesgo sexista como parte del filtro, del marco conceptual o de la grilla en virtud de la cual se ha construido históricamente el saber filosófico como racionalidad masculina? Y, por tanto, ¿no debemos examinar sus condiciones y sus límites? ¿No debemos acaso mostrar también que la dicotomía razón/varón-emoción/mujer siempre ha sido rebasada y solo subsiste como marco prejuicioso de conductas estereotipadas?" (Femenías 2020, p. 27).

Compartimos con la autora que esta dicotomía ha sido rebasada y que siempre ha habido mujeres o disidencias que a lo largo de la historia lograron instalar su voz y producir conocimiento. No obstante, consideramos que no ha sido tan sencillo y que las proporciones fueron y siguen siendo muy desiguales. Podemos pensar en múltiples instancias desde cantidad de autoras mujeres y

Revista Zona Franca- Centro de estudios interdisciplinario sobre las mujeres (CEIM)- Maestría poder y sociedad desde la problemática de género (MG), Rosario, Argentina. ISSN, 2545-6504 http://zonafranca.unr.edu.ar/index.php/ZonaFranca| Número 28 (2020). 
varones, cantidad de publicaciones o incluso cantidad de investigadores en un sistema como el de la Comisión Nacional de Investigaciones Científicas y Técnicas (Conicet), en el que si bien la cantidad de varones y mujeres se ha ido equiparando a lo largo del tiempo, esto sucede sobre todo a nivel de becas o de inicio de la carrera de investigador/a y no sucede de este modo a medida que se asciende en la misma. Múltiples son los análisis que se han realizado al respecto, por lo que podemos pensarlo más como sesgo necesario a asumir y deconstruir que como simple rebasamiento"'!.

\section{Espacios y prácticas emancipadoras para producir}

\section{transformaciones}

La responsabilidad como formadoras de formadoras y formadores y el deseo de transformar, al menos un poco, el tránsito por las instituciones educativas es lo que nos impulsó a abrir espacios en los que la formación docente pudiera empezar a cuestionarse. Hemos realizado tres talleres -en distintos ciclos lectivosen la Facultad de Educación de la UNCuyo en el que todo el estudiantado fue convocado. No había restricción de año o carrera, sino solo las ganas de participar e interrogarse. El primer taller se denominó "Perspectiva de género y educación" y estuvo enfocado en conocer y compartir algunas reflexiones sobre géneros y sexualidades, tal como explicitamos en nuestro marco teórico. Trabajamos pensadoras como Butler, Morgade, Lugones, Korol en un intento de dar a conocer sus aportes y como estos ponían en jaque las subjetividades establecidas. Compartimos selecciones de textos que fuimos analizando en cada encuentro, con una presentación y contextualización previa dada por nosotras. Se inscribieron 40 personas de las cuales 38 eran mujeres, y los 2 varones que empezaron abandonaron luego de 2 encuentros. En los dos talleres siguientes solo se han

III Donna Haraway ha realizado valiosos aportes para pensar este sesgo en la ciencia, y cómo se fue configurando una cierta racionalidad científica masculina de la que se vieron excluidas las mujeres. Su artículo Testigo modesto (2004), entre otros, da cuenta del proceso histórico y epistemológico en el que esto se fue configurando

Revista Zona Franca- Centro de estudios interdisciplinario sobre las mujeres (CEIM)- Maestría poder y sociedad desde la problemática de género (MG), Rosario, Argentina. ISSN, 2545-6504 http://zonafranca.unr.edu.ar/index.php/ZonaFranca| Número 28 (2020). 
inscripto mujeres, y más allá de que la población de la facultad es mayoritariamente femenina, consideramos que esto se ha dado así porque se piensa que el tema de género es un tema exclusivo de las mujeres, de sus reivindicaciones y sus luchas; y no de una sociedad que hay que transformar de manera radical.

Gracias al primer taller observamos que estas temáticas estaban ausentes en la currículalv, que en su mayoría implicaba una gran novedad para nuestras estudiantes y que era un primer paso para comenzar a trabajar estas temáticas ineludibles en el proceso educativo. Con los textos como excusa generamos interrogantes que apuntaban a desandar discursos hegemónicos, que conformaron las subjetividades de nuestras/os estudiantes y que continuarán conformando las de aquellas subjetividades que pasen por sus aulas en tanto no puedan problematizar las mismas. Revisar los preconceptos o espacios del sentido común con el que nos encontramos día a día fue eje de este primer desafío.

Sin embargo cuando armamos el segundo taller decidimos articularlo desde una perspectiva más "práctica" pues estuvo orientado a generar actividades áulicas concretas con perspectiva de género y por ello lo titulamos "Educación en géneros. Problemáticas y propuestas de actividades para trabajar en la escuela", ya que más allá de las posibles explicaciones en términos "teóricos" constatamos que es muy complejo desandar conceptos tan arraigados. El dolor del desaprendizaje que manifiesta Claudia Korol se hace muy patente en estas instancias. Incluso muchas de las estudiantes de este segundo taller ya habían pasado por el primero y al momento de pensar actividades concretas para el aula volvían a reiterarse una y otra vez las nociones hegemónicas y normalizantes de género. Por ello consideramos que no se trataba solo de conocer y comprender teorías feministas -que sin dudas nos ayudan a pensarnos y deconstruirnos- sino

\footnotetext{
IV Luego lo refrendamos con un análisis de la currícula de cada carrera, realizado en un proyecto de investigación dentro de la unidad académica.
}

Revista Zona Franca- Centro de estudios interdisciplinario sobre las mujeres (CEIM)- Maestría poder y sociedad desde la problemática de género (MG), Rosario, Argentina. ISSN, 2545-6504 http://zonafranca.unr.edu.ar/index.php/ZonaFranca| Número 28 (2020). 
de ver qué hacemos con ellas. Cómo trasladamos a las situaciones escolares y cotidianas todas estas rupturas.

Aunque muchas de nuestras estudiantes primero se sorprendían con los interrogantes con los que iniciábamos los talleres, luego comenzaban a comprender los supuestos de sus propias construcciones y subjetividades. No obstante, en muchas instancias tanto de intercambio de opiniones y mucho más aun de producción de actividades, volvían a reiterar sus supuestos, muchas veces incluso justificándolos o apelando a perspectivas de lo normal, lo natural, lo establecido y cómo inclusive eso era parte del fin del sistema escolar. Surgían comentarios del tipo: ¿qué pasaría entonces si cambiamos?, ¿y entonces qué enseñamos ahora? o ¿cómo le enseñamos a los niños "lo que son"? ¿Qué es natural, normal, hasta dónde?, ¿qué van a decirme los directivos, los padres y/o otras docentes?, jseguro voy a tener problemas si hago esto!

Consideramos entonces que más allá de la teoría, que sin duda está a la base y es el marco teórico referencial desde el que nos sostenemos, fue necesario este espacio de taller, apertura y charla para desandar una y otra vez los propios supuestos, a fin de iniciar el largo camino del desaprendizaje. Muchas veces el análisis de diferentes situaciones bajo los mismos conceptos nos resultaba un tanto reiterativo, desde una perspectiva teórico conceptual. Sin embargo, pudimos como docentes también comprender que la experiencia era otra y que se trataba de producir nuevas reiteraciones de discursividades y marcas que puedan no solo repensar algunos conceptos normalizadores, sino también empezar a generar nuevas subjetividades, más libres, más diversas, menos normadas. La performatividad propuesta por Butler jugó aquí un papel central, a modo de resistencia y de la posibilidad de pensar nuevas subjetividades.

Fue un taller de 8 encuentros, uno introductorio y uno de cierre en el las estudiantes presentaron sus aportes. En los 6 restantes armamos actividades para repensar los implementos con los que usualmente trabajan en el aula, a saber,

Revista Zona Franca- Centro de estudios interdisciplinario sobre las mujeres (CEIM)- Maestría poder y sociedad desde la problemática de género (MG), Rosario, Argentina. ISSN, 2545-6504 http://zonafranca.unr.edu.ar/index.php/ZonaFranca| Número 28 (2020). 
uno lo dedicamos a los cuentos, otro a las canciones, otro a los juegos infantiles, el cuarto a los colores y dibujitos, el quinto a las publicidades y el último a las expectativas docentes en función del sexo-género, a saber cómo se esperan aprendizajes diferenciados de acuerdo al género. Como por ejemplo se espera que las nenas sean más prolijas que los varones, que tengan más habilidades manuales, que sean mejores en lengua, mientras que los varones en matemática, educación física y ciencias naturales. Fuimos haciendo preguntas en relación a en qué creían ellas que eran mejores las niñas y en qué los varones y por qué. Las coincidencias fueron abrumadoras y las fundamentaciones hegemónicas. Al momento de revisarlas fue un proceso duro, pero como ya en los encuentros anteriores habíamos logrado desandar muchos preconceptos vinculados a la realidad escolar se dio casi como un cierre necesario. Advirtieron la necesidad de revisar sus propios obstáculos epistemológicos al momento de trasmitir y de evaluar conocimientos, ya que estas marcas generizadas han calado hondo en la subjetividad de todas nosotras.

En los encuentros anteriores pudimos poco a poco visibilizar cuáles han sido los lugares que usualmente se han asignado a las mujeres en los cuentos y las canciones infantiles. La espera, la pasividad, el hogar, la familia, los hijos, la ropa, la limpieza, son las marcas históricas que se siguen reproduciendo en los clásicos infantiles. En relación con los colores, disfraces y juguetes también pudimos reflexionar de manera colectiva, e incluso luego pudieron contar experiencias de sus prácticas docentes en las que vivenciaron la censura de otras maestras al no dejar a un niño disfrazarse con tutú porque "eso es para nenas", o impedirle a las nenas jugar con autitos o herramientas. En el mismo sentido vinculamos esto con las publicidades y el enorme mundo del consumo infantil que segmenta desde el nacimiento de manera binaria. Apostamos a proponer otros juegos, donde nadie quede afuera y donde la clasificación sexual no fuera el centro.

Concluimos el taller con la presentación de actividades prácticas para el aula, con perspectiva de género, a modo de ejercicio performativo en el que las

Revista Zona Franca- Centro de estudios interdisciplinario sobre las mujeres (CEIM)- Maestría poder y sociedad desde la problemática de género (MG), Rosario, Argentina. ISSN, 2545-6504 http://zonafranca.unr.edu.ar/index.php/ZonaFranca| Número 28 (2020). 
estudiantes pudieran visibilizar cuáles son las estrategias posibles y concretas con las que se pueden pensar espacios de libertad que cuestionen la normalización de ellas mismas y de sus futuras/os estudiantes. Trabajaron cuentos clásicos modificándolos, en el que los roles establecidos variaban, o donde se podían visibilizar otras identidades no cisgénero, por ejemplo. Modificaron las letras de canciones infantiles clásicas para irrumpir en los lugares asignados y cambiarlos. Incluso dos estudiantes, que no trabajan en situaciones áulicas sino de consultorio, porque estudian la Licenciatura en Terapia del Lenguaje, pudieron repensar sus materiales cotidianos y lograron modificar diferentes test. Advirtieron que sin alterar el sentido del mismo se podía transmitir otro modo de experimentar los roles de género, puesto que en un test con diferentes figuras cuyo objetivo era que el paciente dijera la palabra "plancha" había una mujer planchando. Poner un hombre planchando parecería muy disruptivo en un principio para ellas, pero habilitaron ese espacio para empezar a percibir otros roles posibles. Estas modificaciones pudieron realizarlas en diferentes figuras del mismo test, en el que lograron identificar identidades normativizadas.

El último taller "Algunas mujeres en la historia: olvido y/o exclusión" -aun en curso- pretende visibilizar a las mujeres dentro de la historia del pensamiento, ya que han quedado siempre fuera del canon. Nos interrogamos acerca de las posibles razones de tal omisión y nos abocamos a traerlas al presente. Analizamos sus vidas y sus aportes y a partir de una selección de textos abrimos rondas de preguntas y comentarios para con el fin de pensar qué de esos aportes pervivieron, cuáles fueron reapropiados por varones y cómo estos se hacen presentes hoy fruto de luchas colectivas. Específicamente nos dedicamos a Hipatia de Alejandría, a Juana Inés de Asbaje, a Olympe de Gouges, Mary Wollstonecraft, Flora Tristán, Simone de Beauvoir y Angela Davis. Un espectro amplio y diverso, pero con el fin de visibilizar como siempre hemos estado presente y aportando al pensamiento y a los derechos humanos de los cuales pretendieron excluirnos.

Revista Zona Franca- Centro de estudios interdisciplinario sobre las mujeres (CEIM)- Maestría poder y sociedad desde la problemática de género (MG), Rosario, Argentina. ISSN, 2545-6504 http://zonafranca.unr.edu.ar/index.php/ZonaFranca| Número 28 (2020). 


\section{Consideraciones finales}

A modo de cierre nos interesa resaltar la importancia de la educación en la construcción de subjetividades más libres. Consideramos ineludible la tarea de pensar qué tipo de educación queremos, cuál impone el sistema y qué nos demandan o presentan las nuevas infancias. Frente a la educación normalizadora sostenida en las bases de una modernidad colonial, capitalista, patriarcal, blanca y heterosexuada, pretendemos generar espacios de conciencia crítica que recalen en prácticas abiertas a la diversidad que encarna cada una/o en cada momento. Abogamos por una escuela abierta, en la que todos los cuerpos tengan un lugar, pero en la que además se sientan cobijados, acompañados y libres de expresar esa utopía de emancipación, que los libere de estigmatizaciones y sesgos. Buscamos que estos talleres despierten al menos una chispa, una duda que irrumpa en lo establecido y dado por supuesto. Pretendemos irrumpir en esas normalidades reiteradas que ocluyen a muchas identidades y muchos cuerpos, muchas veces sin que las mismas docentes puedan percibirlo. Abrir espacios donde puedan preguntar, escuchar, dudar o aun sorprenderse e incluso disentir es parte de nuestra apuesta por la construcción de otra educación posible. Creemos que de a poco esos espacios se han ido consolidando, al menos para que las estudiantes de la Facultad de Educación sepan que existen y junto con ello la posibilidad de pensar juntas otros modos posibles de educar y de vincularse con las infancias.

Consideramos finalmente que se trata de una tarea ardua y cotidiana, la de generar nuevas prácticas que constituyan personas más libres, menos sujetadas, menos normalizadas. La formación docente tiene claras demandas de un mundo cada vez más complejo y siempre diverso. Esperamos aportar a la tarea desde el espacio en el que estamos insertas.

\section{Bibliografía}

Revista Zona Franca- Centro de estudios interdisciplinario sobre las mujeres (CEIM)- Maestría poder y sociedad desde la problemática de género (MG), Rosario, Argentina. ISSN, 2545-6504 http://zonafranca.unr.edu.ar/index.php/ZonaFranca| Número 28 (2020). 
BIDASECA, Karina y VÁZQUEZ LABA, Vanesa (2011). Feminismos y poscolonialidad. Buenos Aires, Ediciones Godot Argentina.

BUTLER, Judith (2002). Cuerpos que importan. Sobre los límites materiales y discursivos del "sexo". Buenos Aires, Paidós.

BUTLER, Judith (2007). El género en disputa. El feminismo y la subversión de la identidad. Barcelona, Paidós.

BUTLER, Judith (2019). Deshacer el género, Buenos Aires, Paidós.

FEMENÍAS, María Luisa (2020). Ellas lo pensaron antes. Filósofas excluidas de la memoria. Buenos Aires, Ediciones Lea.

FOUCAULT, Michel (2009). Historia de la sexualidad 1: la voluntad de saber. Buenos Aires, Siglo Veintiuno Editores.

FOUCAULT, Michel (2010). El cuerpo utópico. Buenos Aires, Nueva Visión.

KOROL, Claudia (2016) Feminismos populares: pedagogías y políticas. Buenos Aires, Editorial Chirimbote.

KOROL, Claudia (2016) "Feminismos populares. Las brujas necesarias en los tiempos de cólera". En: Nueva sociedad. № 265, septiembre-octubre de 2016, p.142-152.

LUGONES, María (2008). "Colonialidad y género". En: Tabula Rasa. Bogotá - Colombia, No.9: 73-101, julio-diciembre. Recuperado de: http://www.revistatabularasa.org/numero-9/05lugones.pdf

MAFFÍA, Diana, comp. (2009). Sexualidades migrantes: género $y$ transgénero. Buenos Aires. Librería de las Mujeres.

MAFFÍA, Diana. "Mujeres: la rebelión que no cesa". Diario La Capital, domingo 3 de marzo de 2019. Recuperado de: https://www.lacapital.com.ar/mas/mujeres-la-rebelion-que-no-cesa-n1741882.html

MANSILLA, Gabriela (2014), Yo nena, yo princesa. Buenos Aires: UNGS.

MANSILLA, Gabriela (2019) "Quiero que el cuerpo travesti y trans esté en la escuela". Página 12. 15/9/19. Recuperado de: https://www.pagina12.com.ar/218304-quiero-que-el-cuerpo-travesti-y-trans-esteen-la-escuela

Revista Zona Franca- Centro de estudios interdisciplinario sobre las mujeres (CEIM)- Maestría poder y sociedad desde la problemática de género (MG), Rosario, Argentina. ISSN, 2545-6504 http://zonafranca.unr.edu.ar/index.php/ZonaFrancal Número 28 (2020). 
MORGADE, Graciela (2001). Aprender a ser mujer, aprender a ser varón. Buenos Aires, Ediciones Novedades Educativas.

MORGADE, Graciela. y ALONSO, Graciela (2008). Cuerpos y sexualidades en la escuela: de la normalidad a la disidencia. Buenos Aires, Paidós.

MORGADE, Graciela, comp. (2011). Toda educación es sexual: hacia una educación sexuada. Buenos Aires, La Crujía.

VÁZQUEZ LABA, Vanesa (2019). Feminismos, género y transgénero: breve historia del siglo XIX hasta nuestros días. Buenos Aires, UNSAM EDITA.

Revista Zona Franca- Centro de estudios interdisciplinario sobre las mujeres (CEIM)- Maestría poder y sociedad desde la problemática de género (MG), Rosario, Argentina. ISSN, 2545-6504 http://zonafranca.unr.edu.ar/index.php/ZonaFrancal Número 28 (2020). 